\title{
A Study of some Cosmological Parameters at Different Eras of the Universe
}

\author{
Mohammed K. Zeki \\ Department of Physics \\ College of Science \\ University of Mosul
}

(Received 17/1/2013 ; Accepted 29/4/2013 )

\begin{abstract}
This work deals with the implication of the concept of the energy density $\rho$ at three different eras (radiation, matter and dark energy dominated eras) through the evolution of the universe. The solution of the Einstein's equations (Friedmann - Robertson - Walker equations, FRW), yields two explicit forms of the scale factor $\mathrm{R}(\mathrm{t})$ : one is the power-law solution for both radiation and matter dominated eras and other is the exponential solution for the dark energy dominated era.

In the present work an equation for the three parameters has been derived, Hubble $(\mathrm{H})$, redshift (z) and deceleration (q), in three different eras, with a thorough discussion.
\end{abstract}

Keywords : FRW space-time, Hubble's and deceleration parameters, redshift, cosmology

\section{درلسة بهض المعاملات الكونيةفي ألرقلبمختلفة الكهن}

\section{ll}

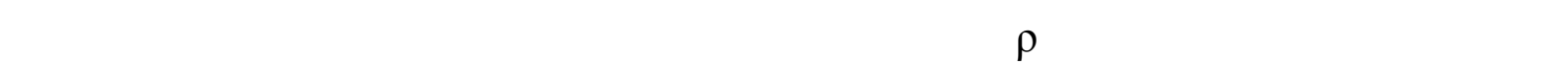

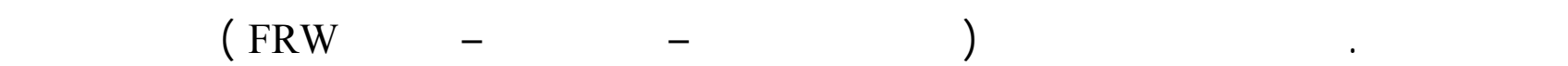
شكلان لأنصف أقطار الكون R(t). الطل الأول تضضمن الرفع إلى القوى للحقبتين الإثبعاعية والمادية والل الثاني أسي (لوغاريتمي) لهقبة الطاقة اللسوداء.

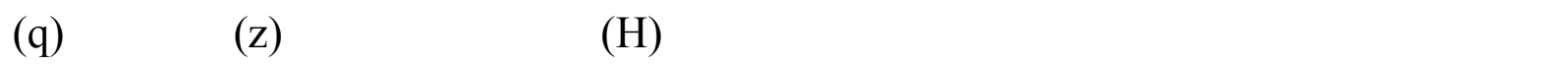
للحقبت الزمنية الثلاثة، وقم منالثة النتائج.

الكامل الدالة: مكان FRW، معلمل هالب، معلمل التبطؤ، الزحزحة الحمراء، كونيت.

\section{INTRODUCTION}

The physical constants are numbers used by scientists in their calculation, which depends on laboratory measurements. The official values of the fundamental constants vary from time to time as new measurements are made. Similarly, the cosmological parameters 
can be assumed to vary and this variation leads to an apparent expansion of the universe, which gives rise to a possibility of explaining some of anomalies found in the observations of high redshift quasars (Kafatos et al., 2005). Present cosmology is based on the Friedmann-Robertson-Walker ( FRW ) model. In this model, the universe is completely homogeneous and isotropic and in agreement with the observational data about the large scale structure of the universe. However, there is no reason to believe in a regular expansion for a description of the early stage of the universe (Verma et al., 2011). But there are theoretical works (Misner, 1968; Chimento, 2004) and recent experimental data of the cosmic microwave background radiation which supports the existence of an anisotropic phase that approaches an isotropic one (Ryan and Shepley, 1975). This stimulates search for exact anisotropic solution of Einstein field equations as cosmologically acceptable physical models for universe at least in its early stage of evolution. There has been a considerable interest in the study of spatially homogeneous and anisotropic cosmological models of Bianchi type I-IX. (Ryan and Shepley, 1975). Bianchi type II space-time plays a fundamental role in constructing models with richer structure both geometrically and physically for describing the early stages of evolution of the universe (Verma et al., 2011). Several works have been done by (Hajj-Boutros, 1985,1986; Shri, R. 1989,1990; Mazumder,1994 ; Camci et al., 2001 ; Pradhan and Kumar, 2001) using different generating techniques (Singh , 2009).

Another solution for the Friedmann-Robertson-Walker field equation was achieved by Berman in 1983 using a law of variation for the Hubble parameter, which yields a constant value of the deceleration parameter, later it has been developed by Berman and Gomide in 1986. In literature, cosmological models with a constant deceleration parameter have been studied by several authors see for example (Singh, 2009).

Edwin Hubble (Hubble, 1929) showed that the more distant the galaxy, the larger the "redshift" in the spectrum. Astronomers traditionally have interpreted the redshift as a Doppler shift induced as the galaxies recede from us within an expanding universe. One of the first indications that there might be a problem with this picture came in the early 1970's. William G., University of Arizona, (Tifft, 1976-1980) noticed a curious and unexpected relationship between galaxy morphological classification (Hubble type), brightness, and redshift. The galaxies in the Coma cluster, for example, seemed to arrange themselves along sloping bands in a redshift against brightness diagram. Moreover, Spirals tended to have higher redshifts than elliptical galaxies. Clusters other than Coma exhibited the same strange relationships. By far the most intriguing result of these initial studies was the suggestion that galaxy redshifts take on preferred or "quantized" value. First revealed in Coma Cluster redshift against brightness diagram, it appeared as if redshifts were in some analogous to the energy levels within the atoms. These discoveries led to the suspicion that a galaxy's redshift may not be related to the Hubble velocity alone. If the redshift is entirely or partially non-Doppler, then it could be an intrinsic property of a galaxy, as basic a characteristic as its mass or luminosity, (Tifft, 1982a, Tifft 1982b; Cocke and Tiff, 1983; Tifft and Cocke, 1987 ; Gribbin,1985; Stewart, 1998).

Finally, one of the most striking and strong evidence discoveries in cosmology is the accelerating universe, this comes through the study of the type Ia supernovae (SN Ia) (Riess, et al., 1998), that the most distant type Ia supernovae are moving away much faster than closer ones suggesting that the universe expansion is actually accelerating and this observed acceleration is due to some new hypothetical energy (dark energy) component with a large 
negative pressure (Riess et al., 2004; Jain and Jhingan, 2010; Kumar, 2010). As a result the deceleration parameter is a good test of the expansion of the universe, such that the universe decelerates when the value of the deceleration parameter is positive whereas it accelerates when the value of the deceleration parameter is negative.

In this paper, an implication of the theoretical basis of the energy density of the universe at three different eras (radiation, matter, and dark energy dominated eras) is found bellow, followed by the solutions of Einstein's field equations which are described by the scale factor. Later, a study of Hubble, redshift and deceleration parameters at the three eras are presented. A brief discussion and conclusion are presented at the last section

\section{ENERGY DENSITY OF THE UNIVERSE}

Starting with homogenous and isotropic universe of energy density $\rho$ (energy (E) per unit volume (V)), and according to the first law of thermodynamic, the energy of the universe (system), (Misner et al., 1973; Vishwakarma and Beesham, 1999; Susskind, 2009), is given by:

$$
\mathrm{E}=\rho \mathrm{V}
$$

The differential form of equation (1) is:

$$
\begin{aligned}
\mathrm{dE} & =\rho \mathrm{dV}+\mathrm{Vd} \rho \\
& =-\mathrm{PdV}
\end{aligned}
$$

where $\mathrm{p}$ is the pressure of the universe, which is given by the equation of state:

$$
\mathrm{P}=\omega \rho
$$

The constant $\omega$ gives the characteristic of the universe at different eras. Substituting equation (3) in equation (2) and integrating yields:

$$
\rho=\frac{\text { cons tan } t}{\mathrm{~V}^{\omega+1}}
$$

Equation (4) describes different values of the energy density at different universal eras depending on the values of $\omega$ as follows:

\section{Energy Density at Radiation (Photon) Dominated Era:}

In this era, the universe is radiation dominated which means there were more photons than matter, and for simplicity take a cubic volume $\left(V=R^{3}(t)\right)$ of space that contains a constant number $(n)$ of photons, where $R(t)$ is the scale factor of the universe. As the universe expands the photon's wavelength expands too (photon inflation), this means that the photon's wavelength is proportional to the scale factor of the universe, then:

$$
\mathrm{E}_{\gamma}=\mathrm{h} v_{\gamma}=\frac{\mathrm{hc}}{\lambda_{\gamma}} \propto \frac{\mathrm{hc}}{\mathrm{R}(\mathrm{t})}=\frac{\mathrm{C}_{\mathrm{r}}}{\mathrm{R}(\mathrm{t})}
$$

where $h$ is Plank's constant, $\mathrm{c}$ is the speed of light, $\mathrm{C}_{\mathrm{r}}\left(\mathrm{C}_{\mathrm{r}}=\mathrm{hc}\right)$ is a constant, finally $v_{\gamma}$ and $\lambda_{\gamma}$ are the frequency and the wavelength of the photon. Then the energy density $\rho_{\mathrm{r}}$ of the radiation dominated reads:

$$
\rho_{r}=\frac{E}{V}=\frac{E}{R^{3}(t)}=\frac{C_{r}}{R^{4}(t)}
$$

Equation (6) can be obtained directly from equation (4). Since in this era the photons can be represented as a system of $n$ particle behaved as a perfect fluid (particles having the same velocity at each point in space, such that an observer in this reference of space $\tilde{X}^{\alpha}$ see 
the fluid around him isotropic, and this reference frame is called commoving coordinate denoted by $\tilde{X}^{\alpha}$ to distinguish it from the ordinary $\mathrm{X}^{\alpha}$, (Weinberg, 1972), such that:

$$
\left.\begin{array}{l}
\tilde{T}^{i j}=p \delta_{i j} \\
\tilde{T}^{i o}=T^{i o}=0 \\
\tilde{T}^{o o}=\rho
\end{array}\right\}
$$

where $\delta_{\mathrm{ij}}$ is the kronical delta $\left(\delta_{\mathrm{ij}}=0\right.$ for $\mathrm{i} \neq \mathrm{j}, \delta_{\mathrm{ij}}=1$ for $\mathrm{i}=\mathrm{j}$ and $\left.\sum_{\mathrm{i}=1}^{3} \delta i i=3, \mathrm{i}=1,2,3\right)$ then:

$$
\mathrm{P} \cong \frac{1}{3} \rho
$$

comparing equations (3) and (8), $\omega=\frac{1}{3}$, then equation (4) becomes:

$$
\rho_{r}=\frac{\text { cons } \tan t}{R^{4}(t)}
$$

Equations (6) and (9) contain the same constants (constant $=\mathrm{C}_{\mathrm{r}}$ ), and both of them describe the energy density of radiation dominated era in which the energy density $\rho_{\mathrm{r}}$ is inversely proportional to the fourth power of the scale factor $\mathrm{R}(\mathrm{t})$.

\section{Energy Density at Matter Dominated Era:}

Universal theories indicate that the universe became a matter dominated somewhere with a few billions of years after the big-bang. Now considering the same procedure of a cubic volume of $R^{3}(t)$ with space containing a constant number of galaxies exerting no outward pressure ( $\mathrm{p}=0$ ), and according to equation (3), $\rho \neq 0$, therefore $\omega=0$, then equation (4) reads:

$$
\rho_{m}=\frac{\text { cons } \tan t}{R^{3}(t)}=\frac{M}{R^{3}(t)}
$$

where $M$ is the mass of the (galaxies) universe, and $\rho_{\mathrm{m}}$ is the energy density for matter dominated era. This equation shows that the energy density $\rho_{\mathrm{m}}$ is inversely proportional to the third power of the scale factor $\mathrm{R}(\mathrm{t})$.

\section{Energy Density at Dark Energy Dominated Era:}

In this era, the astronomical observations show that the universe is an accelerated one, this means that the pressure $P$ in equation (3) is negative, which leads to $\omega=-1$, in which the scale factor is an exponential dependence with time and the energy density becomes very small $\left(\sim 2 \times 10^{-29} \mathrm{gm} / \mathrm{cm}^{3}\right)$ and is referred to as $\rho_{\mathrm{o}}$ (Misner et al.,1973), then equation (4) reads:

$$
\rho_{\mathrm{d}}=\frac{\text { constan } \mathrm{t}}{\mathrm{V}^{\mathrm{o}}}=\text { constan } \mathrm{t}=\rho_{\mathrm{o}}
$$

Here $\rho_{d}$ is the energy density of the dark energy which is also called vacuum energy or the cosmological constant $\Lambda$. 


\section{THE SCALE FACTOR OF THE UNIVERSE}

Starting from the Friedmann-Robertson-Walker equation (Weinberg, 1972):

$$
\frac{\dot{R}^{2}(t)}{R^{2}(t)}-\frac{8 \pi G \rho}{3}=\frac{K}{R^{2}(t)}
$$

where $R(t)$ is the scale factor of the universe, $\rho$ is the total energy density of the three eras $\left(\rho=\rho_{\mathrm{r}}+\rho_{\mathrm{m}}+\rho_{\mathrm{d}}\right), \mathrm{G}$ is Newtonian gravitational constant, and $\mathrm{K}(\mathrm{k}=0, \mathrm{k}=1, \mathrm{k}=-1)$ is the curvature constant for flat, closed and opened universe respectively. The solution of equation (12) gives the scale factor at the three different eras as follows.

\section{The Scale Factor at the Radiation Dominated Era:}

In this era equation (12) can be solved using a guess power law (Mansouri et al., 1999; Susskind, 2009; Zeki et al., 2010):

$$
\begin{aligned}
& \frac{R(t)}{R\left(t_{0}\right)}=\left(\frac{t}{t_{o}}\right)^{\alpha} \\
& R(t)=\frac{R\left(t_{0}\right)}{t_{o}^{\alpha}} t^{\alpha}=C t^{\alpha}
\end{aligned}
$$

where $C=\frac{R\left(t_{0}\right)}{t_{o}^{\alpha}}$, and $\alpha$ are constants. Using equations (9), (12) and (13), and setting $\mathrm{k}=0$ (simple case for flat space), we get :

$$
\frac{\alpha^{2}}{t^{2}}=\frac{8 \pi G C_{r}}{3 C^{4} t^{4 \alpha}}
$$

Setting $\alpha=\frac{1}{2}$ for matching both sides of the above equation then $\mathrm{C}$ can be calculated, and equation (13) yields:

$$
R(t)=\sqrt[4]{\frac{32 \pi G C_{r}}{3}} t^{1 / 2} \quad(\mathrm{t} \text { is small })
$$

Equation (14) shows that the scale factor $\mathrm{R}(\mathrm{t})$ at the radiation dominated era is proportional to the time of power $(1 / 2)$.

\section{The Scale Factor at the Matter Dominated Era :}

In this era, the same procedure can be used to find a relation between the scale factor and time. Using equation (10), (12) and (13), we have :

$$
\frac{\alpha^{2}}{\mathrm{t}^{2}}=\frac{8 \pi \mathrm{GM}}{3 \mathrm{C}^{2} \mathrm{t}^{3 \alpha}}
$$

Setting $\alpha=\frac{2}{3}$ to find the value of $C$, and then equation (13) reads:

$$
R(t)=\sqrt[3]{6 \pi G M} t^{2 / 3}
$$

Equation (15) shows that the scale factor of the universe at the matter dominated era is proportional to the time of power $(2 / 3)$. 
The Scale Factor at the Dark Energy Dominated Era :

In this case the pressure is negative (accelerated universe) and according to equation (11), $\rho_{\mathrm{d}}=\rho_{\mathrm{o}}=$ constant, is a very small quantity $\left(\sim 2 \times 10^{-29} \mathrm{gm} / \mathrm{cm}^{3}\right)$, so equation (12) becomes:

$$
\frac{\dot{R}^{2}(t)}{R^{2}(t)}=\frac{8 \pi G \rho_{o}}{3}
$$

Using the definition of the Hubble parameter (Weinberg, 1972):

$$
\begin{aligned}
& H=H(t)=\frac{\dot{R}(t)}{R(t)} \\
& \text { or } \quad \mathrm{H}=\sqrt{\frac{8 \pi \mathrm{G} \rho_{\mathrm{o}}}{3}} \quad \text { (H is constant) }
\end{aligned}
$$

Now combining equations (13), (16) and (17):

$\ln R(t)=H(t) t+\ln C_{1}$

where $\operatorname{lnC}_{1}$ is the constant of integration which can be calculated using the initial boundary condition at $\mathrm{t}=\mathrm{t}_{\mathrm{o}}, \mathrm{R}(\mathrm{t})=\mathrm{R}\left(\mathrm{t}_{0}\right)$ and $\mathrm{H}=\mathrm{H}_{\mathrm{o}}$, then:

$$
R(t)=C_{1} e^{H(t) t}
$$

where $C_{1}=R_{o} e^{- \text {Ho to }}$, which can be found by astronomical observations. Equation (19) indicates that the scale factor $\mathrm{R}(\mathrm{t})$ of the universe will rapidly exponentially extended describing an accelerated universe (dark energy dominated era).

\section{THE HUBBLE PARAMETER OF THE UNIVERSE}

In this work, we want to study the Hubble parameter at the three different eras, using the definition of Hubble parameter equation (17) as follows:

\section{Hubble Parameter at Radiation Dominated Era:}

In order to find the Hubble parameter in this era, we combine equations (14) and (17) then:

$$
H_{r}(t)=\frac{1}{2} t^{-1}
$$

This equation shows that the age of the universe at the radiation dominated era is half the inverse of the Hubble parameter.

\section{Hubble Parameter at Matter Dominated Era:}

Similarly combining equation (15) and (17) then:

$$
H_{m}(t)=\frac{2}{3} t^{-1}
$$

which shows that the age of the universe at matter dominated era is $\frac{2}{3}$ the inverse of the Hubble parameter. 


\section{Hubble Parameter at Dark Energy Dominated Era:}

In this case, it is clear from equation (18) that the Hubble parameter is constant in this era, because the energy density $\rho_{o}$ is itself constant.

\section{THE COSMOLOGICAL REDSHIFT OF THE UNIVERSE}

Starting from the simple formula for the redshift of a source emitted its radiation at time t (Weinberg, 1972 ; Sciama, 1973).

$\frac{v_{o}}{v}=\frac{t}{t_{o}}=\frac{R(t)}{R\left(t_{0}\right)}$

and

$$
\mathrm{Z}=\frac{\mathrm{v}}{\mathrm{v}_{\mathrm{o}}}-1
$$

where $v_{0}$ and $v$ are the observed and the source frequencies respectively and,

$$
\frac{R(t)}{R\left(t_{0}\right)}=\frac{v_{o}}{v}
$$

then:

$$
R(t)=R\left(t_{0}\right)(1+Z)^{-1}
$$

and it follows (Sandage,1962 ; Samushia and Ratra, 2006 ; Uzan et al., 2008 ; Jain and Jhingan, 2010 ; Yoo et al., 2010; Zeki, 2010).

$$
H(t)=-\dot{Z}(1+Z)^{-1}
$$

The integration of equation (26) gives :

$$
\ln (1+Z)=-H(t) t+\ln C_{2}
$$

where $\ln C_{2}$ is the constant of integration which can be calculated using the initial boundary condition at $\mathrm{t}=\mathrm{t}_{\mathrm{o}}, \mathrm{R}(\mathrm{t})=\mathrm{R}\left(\mathrm{t}_{0}\right)$ and $\mathrm{H}=\mathrm{H}_{\mathrm{o}}$, then the solution of equation (26) is:

$$
\mathrm{Z}=\mathrm{C}_{2} e^{-H(t) t}-1
$$

where $C_{2}=\frac{1+Z_{o}}{e^{-H_{o} t_{o}}}$, which can be found by astronomical observations. Then the redshift at the three different eras are.

\section{Redshift at Radiation Dominated Era :}

Combining equations (20) and (27), then the redshift at the radiation dominated era:

$$
Z_{r}=C_{2} e^{-\frac{1}{2}}-1
$$

\section{Redshift at Matter Dominated Era :}

Similarly combining equation (21) and (27), then the redshift at the matter dominated era:

$$
Z_{m}=C_{2} e^{-\frac{2}{3}}-1
$$

\section{Redshift at Dark Energy Dominated Era :}

The same procedure can be used by combining equation (18) and (27), we get the redshift at the dark energy dominated era:

$$
\mathrm{Z}_{\mathrm{d}}=\mathrm{C}_{2} \mathrm{e}^{-\sqrt{\frac{8 \pi \mathrm{G} \rho_{\mathrm{o}}}{3}} \mathrm{t}}-1
$$


Equation (30) shows that the redshift is time dependent.

\section{THE DECELERATION PARAMETER OF THE UNIVERSE}

In this case, we want to find the values of the deceleration parameter in the three different eras using the well-known definition of the deceleration parameter (Weinberg, 1972).

$$
q_{(t)}=q=-\frac{\ddot{R}(t)}{\dot{R}(t)} \frac{R(t)}{\dot{R}(t)}
$$

\section{Deceleration at Radiation Dominated Era :}

Differentiate equation (14) twice with respect to time:

$$
\dot{R}(t)=\frac{1}{2} \sqrt[4]{\frac{32 \pi G C_{r}}{3}} t^{-\frac{1}{2}}
$$

and

$$
\ddot{R}(t)=-\frac{1}{4} \sqrt[4]{\frac{32 \pi G C_{r}}{3}} t^{-3 / 2}
$$

Then substitute equations (14), (32) and (33) in equation (31), then the deceleration parameter at the radiation dominated era is:

$$
\mathrm{q}=+1
$$

\section{Deceleration Parameter at Matter Dominated Era :}

In order to find the deceleration parameter at the matter dominated era, we differentiate equation (15) twice with respect to time:

$$
\begin{aligned}
& \dot{R}(t)=\frac{2}{3} \sqrt[3]{6 \pi G M} t^{-1 / 3} \\
& \ddot{R}(t)=\frac{-2}{9} \sqrt[3]{6 \pi G M} t^{-4 / 3}
\end{aligned}
$$

Substitute equations (15), (35) and (36) in equation (31), then:

$$
q=\frac{1}{2}
$$

Equation (34) and (37) show that the value of q is greater than zero, then in these two eras the universe decelerates

\section{Deceleration Parameter at Dark Energy Dominated:}

Again the same procedure can be used by differentiating equation (19) twice with respect to time, we have:

$$
\begin{aligned}
& \dot{R}(t)=C_{1} H e^{H(t) t} \\
& \ddot{R}(t)=C_{1} H^{2} e^{H(t) t}
\end{aligned}
$$

Substituting equations (19), (38) and (39) in equation (31), then the deceleration parameter at the dark energy reads: 
$\mathrm{q}=-1$

Equation (40) shows that the universe has a deceleration parameter less than zero, which means that the universe accelerates

\section{DISCUSSION AND CONCLUSION}

Starting with homogenous and isotropic universe, we have presented the energy density $\rho$ of the universe equation (4) for different universal eras depending on the value of $\omega$, which represents the characteristic of the universe $(\omega=1 / 3$ for radiation dominated era, $\omega$ $=0$ for matter dominated and $\omega=-1$ for the dark energy dominated era).

A class of solutions to Einstein's equations (FRW equations) is presented. The power law solution equation (13), was used for the three different eras. For the radiation era, it is clear from equation (14) $\left(\mathrm{R}(\mathrm{t}) \alpha \mathrm{t}^{1 / 2}\right)$ that the scale factor $\mathrm{R}(\mathrm{t})$ represents a small, hot and opaque universe (early universe), which is dominated by radiation, i.e. the majority of the energy is in the form of photons and some massless particles, with energy density given by equation (9), ( $\left.\rho_{\mathrm{r}} \alpha \mathrm{R}^{-4}(\mathrm{t})\right)$. Later, the universe cools down due to the expansion and entered a mass dominated era. The scale factor is given by equation $(15),\left(R(t) \alpha t^{2 / 3}\right)$, with energy density equation (10), $\left(\rho_{\mathrm{m}} \alpha \mathrm{R}^{-3}(\mathrm{t})\right)$.

At larger values of $t$, the dark energy becomes dominated (equation 19) causing an accelerating expansion, such that the universe is rapidly extended (inflation) in a time rate of change exponentially with constant energy density $\rho_{0}$ equation (11) and with negative pressure equations (3), giving an accelerated universe. It can be concluded that the universe always decelerated unless its pressure is negative.

Observations also suggested that there had been a transition of the universe from the earlier deceleration phase to the recent acceleration phase, the cause of this sudden transition and the source of the accelerated expansion is still unknown (Kumar, 2010).

In the next step, it is clear from equation (20) that time dependence of the Hubble parameter in the radiation era is $\frac{3}{4}$ relative to that in the matter dominated era (equation 21), while the Hubble parameter (equation 18) at dark energy dominated era seems to be constant. Our conclusion is that these equations measure the dynamics of the universe directly through the Hubble expansion factor. Furthermore, it assumes that the universe is homogenous and isotropic.

The Hubble parameter (equation 26), in terms of the time drift $\dot{z}=\frac{d z}{d t}$ of the cosmological redshift z (Smaushia and Ratra, 2006 ; Uzan et al., 2008 ; Yoo et al., 2010 ; Jain and Jhingan, 2010 ; Zeki et al., 2010), leads to the general form of the cosmological redshift $\mathrm{z}$ (equation 27), such that the cosmological redshift $\mathrm{z}$ vary as a time rate of change exponentially with the Hubble parameter $\mathrm{H}(\mathrm{t})$. This equation describes the redshift at the three different eras (equations 28, 29 and 30) depending on the Hubble parameter $\mathrm{H}(\mathrm{t})$ for each era and on the constant $\mathrm{C}_{2}$, which can be obtained from the astronomical observations. The conclusion is that the variation of the cosmological redshift parameter $\mathrm{z}$, makes it as a good test which maps the expansion of the universe. Moreover, it is clear that $\dot{z}$ in equation (26) is also a good indicator which directly measures the expansion rate of the universe, 
such that, when $\dot{z}$ is less than zero, yields a decelerated universe, while when $\dot{z}$ is greater than zero yields an accelerated universe (Jain and Jhingan, 2010).

Now equations $(34,37$ and 40$)$ show that the deceleration parameters vary at the three different eras. It is clear from equation (34) and (37), that the universe undergoes a decelerate expansion in both radiation and matter dominated eras, the reason is that $\mathrm{q}_{\mathrm{r}}$ and $\mathrm{q}_{\mathrm{m}}$ are greater than zero, while equation (40) shows an accelerating expansion, because the value of $\mathrm{q}_{\mathrm{d}}$ is less than zero. It is generally believed that this acceleration is due to some sort of dark energy is pervading the whole universe (Singh, 2009; Tripathi et al., 2011; Verma et al., 2011 ; Akarsu and Dereli, 2012). SN Ia survey, weak lensing (Heavens, 2003), and baryon acoustic oscillation (BAO) in the galaxy power spectrum (Seo and Eisenstein, 2003; Rudd et al., 2008) are all generally considered to be excellent probes of the acceleration of the universe (Liske et al., 2008a ; Liske et al., 2008b). Our conclusion is that the deceleration parameter $\mathrm{q}^{\prime} \mathrm{s}$ is a good tool for probing the expansion of the universe, because it is reasonable for a description of different phases of the universe. There had been a transition of the universe from earlier deceleration phase to recent acceleration phase, depending on the value of the deceleration parameter, such that the universe decelerates when the value of $q$ is positive, whereas it accelerates when the value of $q$ is negative with negative pressure. Moreover, the universe has an expansion with constant rate if the deceleration parameter q equals zero (Jain and Jhingan, 2010; Cai and Tue, 2012).

Finally, this work shows that the Hubble, redshift and deceleration parameters which are derived at radiation, matter and dark energy dominated eras are no longer constants, but each of them has its own characteristic value at each era.

\section{REFERENCES}

Akarsu, O.; Dereli, T. (2012). Cosmological models with linearly varying deceleration parameter. Int. J. Theor. Phys., 51, 612.

Berman, M.S. (1983). A special law of variation for Hubble's parameter. Nuovo Cimento B. 74, 182-186.

Berman, M.S.; Gomide, F.M. (1986). Cosmological models with constant deceleration parameter. General Relativity and Gravitation. 20, 191-198.

Cai, R-G.; Tue, Z-L. (2012). Direction dependence of the deceleration parameter. arXiv: $1109.0941 v 6$ [astro-ph.co].

Camci, U.; Yavuz, I.; Baysal, H.; Tarhan, I.; Yilmaz, I. (2001). Generation of Bianchi typeV universes filled with a perfect fluid. Astrophs. Space Sci., 275( 4), 391.

Chimento, L.P. (2004). Extended tachyon field, chaplygin gas and solvable k-essence cosmologies. Phys. Rev., D69(12), id 123517.

Cocke, W.J.; Tifft, W.G. (1983). Redshift quantization in compact group of galaxies. Astrophysics letters 23, 239; Astrophysical J., 288, 22.

Gribbin, J. (1985). Galaxy redshift come in clumps. New Scientist, 20-21.

Hajj-Boutros, J. (1985). On spherically symmetric perfect fluid solution. J. Math. Phys., 26, (4), 2297.

Hajj-Boutros, J. (1986). New cosmological models. Class. Quant Grav. 3, 311.

Heavens, A. (2003). 3-D weak lensing, Mon. Not. Roy Astron. Soc., 343, 1327-1334.

Hubble, E. (1929). A relation between distance and radial velocity among extra-galactic Nebula. Proc. Nat. Acad. Sci., (USA) 15, 168-173. 
Jain, D.; Jhingan, S. (2010). Constraints on dark energy and modified gravity models by cosmological redshift drift test. arXiv: 0910.4825v5[gr.qc].

Kafatos, M.; Roy, S.; Roy, M. (2005). Variation of physical constant, redshift and the arrow of time. Acta Physica Polonica B, 36, No. 10.

Kumar, S. (2010). Some FRW models of accelerating universe with dark energy. arXiv: 10100672v1 [gr-qe].

Liske, J.; Grazian, A.; Vanzella, E.; Dessauges, M.; Viel, M.; Pasquini, L.; Haehnelt M.; Gristiani S.; Pepe, F.; Avila, G.; Bonifacio, P.; Bouchy, F.; Dekker, H.; Delabre, B.; D'odorico, V.; Levshakov, S.; Lovis, C.; Mayor, M.; Molaro, P.; Moscardini, L.; Murphy, M.T.; Queloz, D.; Shaver, P.; Udry, S.; Wiklind T.; Zucker S. (2008a). Cosmic dynamics in the eras of extremely large telescopes. Mon. Not. Roy. Astron. Soc. 386, 1192-1218. arXiv: 0802. 1532v1 [ astro-phy ].

Liske, J.; Grazian, A.; Vanzella, E.; Dessauges, M.; Viel, M.; Pasquini, L.; Haehnelt, M.; Gristiani, S.; Pepe, F.; Avila G.; Bonifacio, P.; Bouchy, F.; Dekker, H.; Delabre B.; D'odoric, V.; Levshakov, S.; Lovis, C.; Mayor, M.; Molaro, P.; Moscardini, L.; Murphy, M.T.; Queloz, D.; Shaver, P.; Udry, S.; Wiklind, T.; Zucker, S. (2008b). E-ELT and the cosmic expansion history- A far stretch ). The messenger, 133.

Mansouri, R.; Nasseri, F.; Khorrami, M. (1999). Effective time variation of G in a model universe with variable space dimension. Physics letter A, 259, 194-200.

Mazumder, A. (1994). Bianchi I space-time filled with a perfect fluid. Gen. Relativ. Gravit. 26(3), 307.

Misner, C.W. (1968). The isotropy of the universe. Astrophysical J., 151, 431.

Misner, C.W.; Thorne, K.S.; Wheeler, J.A. (1973) "Gravitation". Freeman and Company, pp. 559-726.

Pradhan, A.; Kumar, A. (2001). LRS Bianchi I cosmological universe models with varying cosmological term. Int. J. Mod. Phys. D10, 291.

Riess, A.G.; Filippenko, A.V.; Challis, P.; Clocchiatti, A.; Diercks, A.; Garnavich, P.M.; Gilliland, R.I.; Hogan, C.J.; Jah, A.; Kirshner, R.P.; Leibundgut, B.; Phillips, M. M.; Reiss, D.; Schmidt, B.P.; Schommer, R.A.; Chris smith, R.; Spyromilio, J.; Stubbs, C.; Suntzeff, N.B.; Tonry, J. (1998). Observational evidence from supernovae for an accelerating universe and cosmological constant. The Astronomical J., 116, 1009-1038, arXiv: astro-phy $19805201 \mathrm{v1.}$

Riess, R.G.; Sirolger, L.C.; Torny, J. (2004). Type Ia supernova discoveries at z $>1$ from the Hubble space telescope: Evidence part deceleration and constraints on dark energy evolution. Astrophysics J., 607, 665-682.

Rudd, D.H.; Zentner, A.R.; Kravtsov, A.V. (2008). Effect of baryons and dissipation on the matter power spectrum. The Astrophysical J., 672, 19.

Ryan, M.P.; Shepley, L.C. (1975). "Homogeneous Relativistic Cosmologies". (Princeton University press, Princeton-New Jersey).

Samushia, L.; Ratra, B. (2006). Cosmological constraints from Hubble parameter versus redshift data: arXiv: astro-ph / 0607301v2.

Sandage, A. (1962). The change of redshift and apparent Luminosity of galaxies due to the deceleration of selected expanding universe. APJ, 136, 3195.

Sciama, D.W. (1973). "Modern Cosmology". Cambridge University Press. 
Seo, H-J.; Eisenstein, D.J. (2003). Probing dark energy with baryonic acoustic oscillations from future large galaxy redshift surveys. Astro. Phys. J. 598, 720-740: arXiv: astro-phy/0307460.

Shri, R. (1989). Generation of LRS Bianchi type-I universe filled with perfect fluids. Gen. Relativ. Gravit. 21(7), 697.

Shri, R. (1990). Bianchi type-V perfect fluid space-time Int. J. Theor. Phys. 29( 8), 901.

Singh, C.P. (2009) Locally-rotationally-symmetric Bianchi type-V cosmology in general relativity. Pramana, journal of physics 72(2), 429-443.

Stewart, M. (1998). On the quantization of the red-shifted light from distant galaxies: tiffshift.html http://www.ldolphin.org/

Susskind, L. (2009). Documents.

Tifft, W.G. (1976). Discrete states of redshift and galaxy dynamics I. Astrophysical J., 206, 38-56.

Tifft, W.G. (1977a). Discrete states of redshift and galaxy dynamics II: Systems of galaxies. Astrophysical J., 211, 31-46.

Tifft, W.G. (1977b). Discrete states of Redshift and galaxy dynamics III: Abnormal galaxies. Astrophysical J., 211, 377-391.

Tifft, W.G. (1978). The discrete redshift and asymmetry in HI profiles. Astrophysical J., 221, 449-455.

Tifft, W.G. (1979). Structure within redshift-magnitude band. Astrophysical J. 233, 799-808

Tifft, W.G. (1980). Periodicity in the redshift intervals for double galaxies. Astrophysical J., 236,70-74.

Tifft W.G. (1982a). Double galaxy investigation II. Astrophysical J., 262, 44-47.

Tifft, W.G. (1982b). Quantum effects in the redshift intervals for double galaxies. Astrophysical J., 257, 442-499.

Tifft, W.G. ; Cocke, W. J. (1987). Quantized Galaxy redshift. Sky an Telescope Magazine, 19-21.

Tripathi, S.K.; Dubey, R.K. (2011). FRW cosmological model of the universe and deceleration parameter. Indian J. Sci. 2(4), 95-98.

Uzan, J-P.; Clarkson, C.; Ellis, F.R. (2008). Time drift of cosmological redshift as a test of the Copernican principle. arXiv: 0801.0068v2 [astro-ph].

Verma, M. K.; Zeyauddin, M.; Ram, S. (2011). Anisotropic cosmological model with negative constant deceleration parameter and time-decaying term. Rom. J. Phys., V 56( 3-4), 616-626.

Vishwakarma, R.G. ; Beesham, A. (1999). RW models with variable G and constant active gravitational mass. Il Nuovo Cimento, 114 B (6).

Weinberg, S. (1972)." Gravitation and Cosmology". John Wily and Sons. Inc., New York.

Yoo, C.; Kai, T.; Nakao, K. (2010). Redshift drift in LTB void Universes. arXiv: 1010.0091v1[astro- phy co.] .

Zeki, M. K.; Doad, N.A.; Abdel-Kadder, A. J. (2010). A new approach for evaluation of the Hubble and the deceleration parameter. J. Rafidain Sci., 12(4). 